J. Lake Sci. (湖泊科学), 2015, 27(4):719-726

DOI $10.18307 / 2015.0421$

(c) 2015 by Journal of Lake Sciences

\title{
鄱阳湖越冬水鸟栖息地评价
}

\author{
夏少霞 ${ }^{1,3}$, 刘观华 $^{2}$, 于秀波 ${ }^{* * *}$, 刘 宇 ${ }^{1}$ \\ (1:中国科学院地理科学与资源研究所生态系统网络观测与模拟重点实验室, 北京 100101) \\ (2: 鄱阳湖国家级自然保护区管理局,南昌 330038) \\ (3:中国科学院大学, 北京 100049)
}

摘 要: 参照国际重要鸟区的评定标准,建立基于种群数量综合因子、物种多样性因子、保护物种综合因子的水鸟栖息地 重要性评价因子集,采用模糊综合法评估鄱阳湖 62 个子湖泊作为越冬水鸟栖息地的重要性.结果表明: (1) 鄱阳湖的水 鸟栖息地总体处于比较重要的水平,子湖泊之间栖息地的重要性呈现出明显的空间差异,自然保护区内高于保护区外; (2) 不同子湖泊间栖息地重要性的变化趋势不同, $53.23 \%$ 的子湖泊栖息地重要性上升, $46.77 \%$ 的子湖泊栖息地重要性 下降,自然保护区内重要性下降的子湖泊比重低于自然保护区外. 因此,当前保护区对栖息地的保护和管理是有效的,但 省级以下保护区内的一些子湖泊的重要性存在下降的趋势,当前仍存在部分保护空缺. 本研究为水鸟栖息地的保护与管 理提供了科学支撑.

关键词: 鄱阳湖;水鸟栖息地;模糊综合评价法;栖息地重要性

\section{Importance assessment of wintering habitats for migratory waterfowl in Lake Poyang}

\author{
XIA Shaoxia ${ }^{1,3}$, LIU Guanhua ${ }^{2}$, YU Xiubo ${ }^{1} \&$ LIU Yu ${ }^{1}$ \\ (1: Key Laboratory of Ecosystem Network Observation and Modeling, Institute of Geographic Science and Natural Resources \\ Research, Chinese Academy of Sciences, Beijing 100101, P. R. China) \\ (2: Jiangxi Poyang Lake National Nature Reserve, Nanchang 330038, P. R. China) \\ (3: University of Chinese Academy of Sciences, Beijing 100049, P. R. China)
}

Abstract: Lake Poyang is the most important over-wintering and staging site for waterfowl along the East Asia-Australasia Flyway. In recognition of its global importance, Lake Poyang was designated a Ramsar site(Wetlands of International Importance) in 1992 for the lake is also home to 300000 to 700000 over-wintering migratory birds. In regards to the rating criteria of important bird area, a factor set is established based on the multi-stress of species, number, biodiversity, as well as protected birds. The fuzzy synthetic method is used to evaluate importance grade of the waterfowl habitat. Spatial pattern and annual variation of 62 sub-lakes are analyzed by using the waterfowl survey data from 2001 to 2013. Management effectiveness of protected areas is also evaluated. Results show that: (1) Waterfowl habitats in Lake Poyang as a whole is in the important grade, however, there is spatial heterogeneity of importance grade of the waterfowl habitatat sub-lake level. (2) Sub-lakes differ in inter-annual variability, with 53. 23\% of the sub-lakes showing an increasing trend, and the others decreasing, however, decrease rate of sub-lakes within natural reserves is lower than those outside. Meanwhile, it is also indicated there are conservation gaps. Sub-lakes such as Lake Zhu and Lake Qi, which are outside the protection areas, are of high importance grade and require better protection. Generally, this study suggests that the current habitat protection and management is effective, nevertheless, the importance grade of some sub-lakes within the provincial protection level shows a downward trend. Hydrological dynamics, high intensity aquaculture and the unclear ownership of sub-lakes within protection areas below provincial level may contribute to changes of importance grade of sub-lakes. This study provides scientific support to waterfowl habitat conservation and management.

Keywords: Lake Poyang; habitat of waterfowl; fuzzy synthetic method; importance grade

* 国家自然科学基金项目 (41171030)资助. 2014-08-04 收稿;2014-12-01 收修改稿. 夏少霞 (1983 ),女,博士 研究生;E-mail:xiasx@ igsnrr. ac.cn.

** 通信作者;E-mail:yuxb@ igsnrr. ac. cn. 
鄱阳湖是东亚一澳大利西亚候鸟迁徙路线上最重要的越冬地和停歇地,每年有 30 万至 70 万只水鸟在鄱 阳湖越冬,其栖息地保护对整个候鸟迁徙路线保护具有重要意义. 近年来,鄱阳湖淡水养殖面积呈现逐年增 加的趋势, 2000-2011 年, 淡水养殖面积增加了 27\% ${ }^{[1]}$. 高强度的水产养殖带来水生植被退化、湿地生态功 能减退等多种负面影响, 导致水鸟栖息地质量明显衰退, 其生存空间也在不断缩小 ${ }^{[2-3]}$. 在经济发展与生态 保护的权衡中,栖息地功能更高、更脆弱的水鸟栖息地应得到优先保护,这将有利于提高生物多样性的保护 效率, 因此对栖息地重要性进行评价,识别出水鸟栖息地优先保护区显得尤为重要 ${ }^{[4-5]}$.

根据《湿地公约》的定义,水鸟是指在生态上依赖于湿地,且形态和行为上对湿地形成适应特征的鸟类. 一方面, 水鸟是湿地生态系统的重要组成部分, 位于湿地生态系统的食物链顶端, 在湿地生态系统能量流动 和维持生态系统稳定性方面起着重要作用 ${ }^{[6-7]}$; 另一方面, 水鸟对湿地生境质量及其结构变化极为敏感, 其 分布、数量、多样性等特征的变化在一定程度上也与湿地面积、植被丰度、水深、景观指数等环境因子密切相

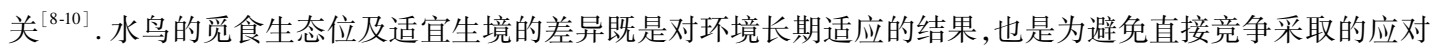
策略 ${ }^{[11-12]}$. 因此, 水鸟群落结构的变化往往反映了湿地不同营养层次的变化 ${ }^{[13-14]}$. 湿地环境中水鸟的群落结 构及丰度被认为是监测湿地变化状况、评价湿地恢复程度的重要指标之一 ${ }^{[15-17]}$. 反映水鸟群落结构及丰度 变化的因子众多,利用单一因子识别栖息地重要性的结果往往不够准确,而采用综合性的因子是进行栖息 地优先性评判的一种有效手段 ${ }^{[18]}$. 本文利用 2000/2001-2012/2013 年连续 13 年的水鸟调查数据, 采用综 合评价法对鄱阳湖水鸟栖息地重要性进行评价,识别了鄱阳湖不同子湖泊作为越冬水鸟栖息地的重要性及 其变化趋势.

\section{1 研究区概况}

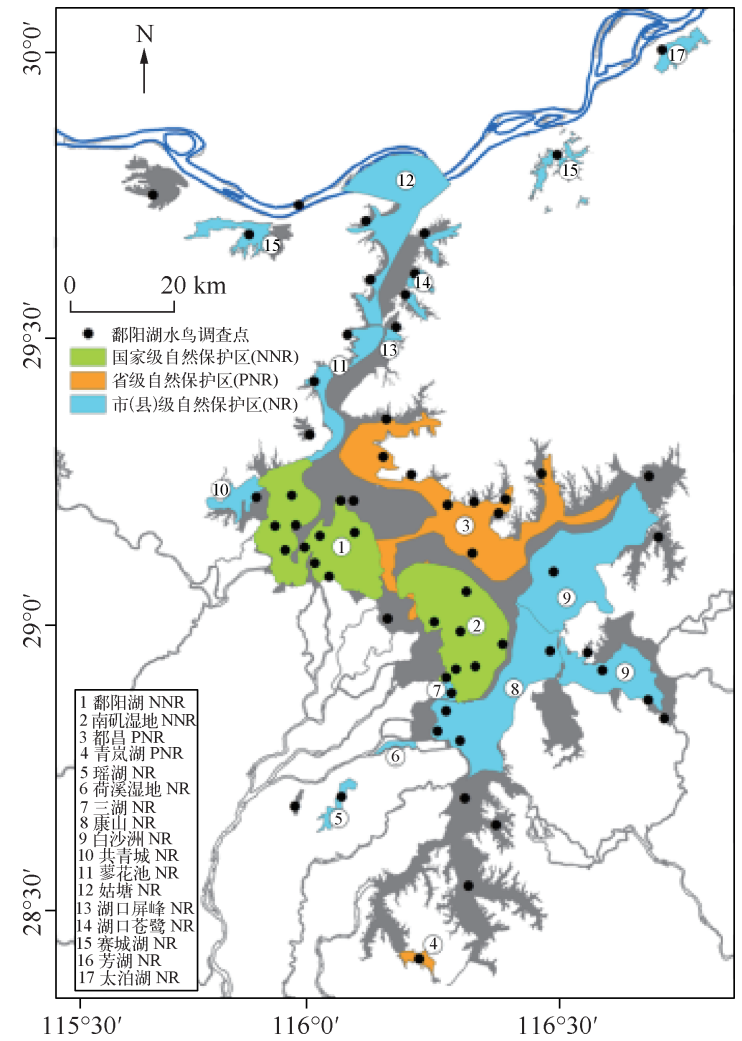

图 1 研究区位置

Fig. 1 Location of study area
鄱阳湖 $\left(28^{\circ} 22^{\prime} \sim 29^{\circ} 45^{\prime} \mathrm{N}, 115^{\circ} 47^{\prime} \sim 116^{\circ} 45^{\prime} \mathrm{E}\right)$ 是中国第一大淡水湖泊, 位于江西省北部, 长江 中下游交接处的南岸. 其丰、枯水期水位变幅明 显, 丰水期湖面面积最高可达 $3600 \mathrm{~km}^{2}$ 以上, 枯 水期湖面面积低于 $1000 \mathrm{~km}^{2[19-20]}$. 枯水期, “水 落滩出” 形成众多与主湖隔离的浅碟型湖, 是越 冬候鸟的主要栖息地 ${ }^{[21]}$. 根据水鸟调查的位置 信息, 本研究将鄱阳湖划分为 62 个子湖泊. 目 前, 在鄱阳湖湿地与候鸟相关的自然保护区共 17 个,其中国家级自然保护区 2 个, 保护面积为 $55700 \mathrm{hm}^{2}$; 省级自然保护区 2 个, 保护面积为 $42100 \mathrm{hm}^{2}$; 市 (县) 级自然保护区共有 13 个, 保 护区面积为 $98817 \mathrm{hm}^{2} .17$ 个不同保护级别的保 护区共覆盖了 62 个子湖泊中的 30 个, 占子湖泊 总面积的 $52.23 \%$ (图 1).

\section{2 数据与研究方法}

\section{1 数据来源}

本文以 2000/2001－2012/2013 年越冬季鄱 阳湖水鸟同步调查数据为主要数据源, 于每年 的 12 月至次年 2 月进行. 该时间段水鸟集中在 鄱阳湖越冬, 数量相对稳定, 水鸟占据了最佳栖 息地. 对水鸟的统计采用直接计数和集团计数 2 种方法 ${ }^{[22]}$, 鸟类的识别和鉴定参考《中国鸟类野 外手册》 ${ }^{[23]}$. 


\section{2 研究方法}

本文采用模糊综合评价法对 62 个子湖泊作为越冬水鸟栖息地的重要性进行评价,并分析各子湖泊栖 息地重要性的变化趋势. 模糊综合评判方法是一种基于模糊数学的综合评标方法,基于模糊数学的隶属度 理论将定性评价转化为定量评价. 一般依据给定的评价标准和评价因子的实测值,经过模糊变化,给每个评 价因子赋予一个非负实数,得到评价结果, 再与评语集相对照. 该方法常用于环境质量评价、环境影响评价 中 $^{[24-25]}$. 近年来, 又逐渐被引人水鸟栖息地重要性、适宜性、保护空缺等的评价中 ${ }^{[18,26]}$.

2.2.1 评价因子的确定 目前, 评价水鸟栖息地质量及变化状况的综合因子很多,如以群落的丰富度、地理 分布和栖息地选择性为基础提出的群落保护指数 ( community conservation index $)^{[27]}$; 基于物种濒危等级提出 的 IUCN 红色名录指数 (red list index) ${ }^{[28]}$; 国际鸟盟 (Bird Life International) 提出的重要鸟区 (important birds area, IBA) 的划分标准 ${ }^{[29]}$ 以及基于水鸟的种类、数量、生态类群及稀有性提出的综合指数 ${ }^{[18]}$ 等. 本文在上述 因子的基础上,篮选了水鸟数量种数、物种多样性及珍稀水鸟的数量和种类等指示水鸟群落变化的核心因 子,构建了鄱阳湖水鸟栖息地重要性评价综合因子集: $U=\{A, B, C\}$. 其中, $A$ 为各子湖泊中水鸟的种类和数 量综合因子; $B$ 为各子湖泊中物种多样性因子, 此处采用 Shannon-Wiener 指数为评价指标; $C$ 为各子湖泊中 保护物种的种类、数量综合因子 (种类、数量均按换算后的国家一级物种计算, 1 个国家一级物种按 2 个二级 保护物种计算, 列人 IUCN 红色物种名录但未列入国家一级和二级重点保护物种的视为国家二级重点保护 物种).

根据水鸟调查数据, 分别计算 $2000 / 2001-2012 / 2013$ 年各子湖泊水鸟的种类、数量、物种多样性指数以 及保护物种种数和数量等指标, 获 得 $A 、 B 、 C 3$ 个评价因子的原始数 据. 其中 Shannon-Wiener 指数计算 公式为: $H^{\prime}=-\sum_{i=1}^{n} P_{i} \cdot \ln P_{i}$, 其 中: $P_{i}=n_{i} / N$, 式中: $n$ 为调查子湖泊 中水鸟的种数, $n_{i}$ 为第 $i$ 个种水鸟的 个体数, $N$ 为调查湖泊中所有水鸟 的个体总数. $A 、 B 、 C 3$ 个评价因子的 值参照表 1 , 将 3 个评价因子的原始 数据根据隶属函数转化为相应 的值.

2.2.2 隶属函数的建立 各因子的 最佳值参考国际鸟盟的 IBA 评定标 准 ${ }^{[29]}$. 最佳值及其以上值的隶属函 数值为 1 , 据此建立各因子的隶属函 数 $U(x)$, 如表 2 所示.

2.2.3 评价因子权重的确定对因 子权重采用逐项比较的方法, 即对 3 个评价因子逐项就任意 2 个评价因 子对栖息地的影响大小进行比较, 重要程度远大于另一个因子时,给 3 表 1 评价因子的权重 Tab. 1 Weight of evaluation factors

\begin{tabular}{cccccc}
\hline 评价因子 & $A$ & $B$ & $C$ & 总分 & 权重值 \\
\hline$A$ & - & 1 & 1 & 2 & 0.2 \\
$B$ & 2 & - & 1 & 3 & 0.3 \\
$C$ & 3 & 2 & - & 5 & 0.5 \\
\hline
\end{tabular}

表 2 各评价因子的隶属函数

Tab. 2 Subject function of evaluation factors

\begin{tabular}{|c|c|c|}
\hline 评价因子 & 因子最佳值 & 隶属函数 \\
\hline 种类 $A_{t}$ & 20 & $U\left(A_{t}\right)\left\{\begin{array}{l}A_{t} / 20,0 \leqslant A_{t}<20 \\
1, A_{t} \geqslant 20\end{array}\right.$ \\
\hline 数量 $A_{n}$ & 20000 & $U\left(A_{n}\right)\left\{\begin{array}{l}A_{n} / 20000,0 \leqslant A_{n}<20000 \\
1, A_{n} \geqslant 20000\end{array}\right.$ \\
\hline 物种多样性指数 $B$ & $B_{\max }$ & $U\left(B_{t}\right)\left\{\begin{array}{l}B_{t} / B_{\max }, B_{t}<B_{\max } \\
1, B_{t}=B_{\max }\end{array}\right.$ \\
\hline 保护物种种数 $C_{t}$ & 4 & $U\left(C_{t}\right)\left\{\begin{array}{l}C_{t} / 4,0 \leqslant C_{t}<4 \\
1, C_{t} \geqslant 4\end{array}\right.$ \\
\hline 保护物种数量 $C_{n}$ & 400 & $U\left(C_{n}\right)\left\{\begin{array}{l}C_{n} / 400,0 \leqslant C_{n}<400 \\
1, C_{n} \geqslant 400\end{array}\right.$ \\
\hline
\end{tabular}
分, 相对重要者给 2 分, 不重要者给

1 分; 每一评价因子得分之和与各评价因子得分之和的比值即为该评价因子的权重 ${ }^{[30]}$. 因子权重确定过程 见表 1 , 根据权重比较结果, 权重排序为 $C>B>A$. 评价因子的权重集 $W$ 为: $W=\{C, B, A\}(0.5,0.3,0.2)$. 此 外, $A$ 和 $C$ 由种类数与个体数 2 个次级因子所组成, 根据物种多样性的原则规定种类数的权重值为 0.6 , 个 体数量的权重值为 $0.4^{[18]}$. 最后, 结合评价因子的权重, 最终得到水鸟栖息地功能重要性指数, 本文以 2000/ $2001-2012 / 2013$ 年的平均值作为各栖息地功能重要性指数. 


\section{3 研究结果}

\section{1 鄱阳湖水鸟数量、种类、多样性及其变化}

2000/2001-2012/2013 年越冬季共调查到国家一级重点保护水鸟 5 种, 国家二级重点保护水鸟 11 种, 列人 IUCN 红色物种名录的水鸟 13 种. 其中, 大湖池、珠湖、蚌湖、沙湖、大汉湖的水鸟平均数量达到 20000 只以上, 大湖池、大汉湖、蛙湖、赛城湖、珠湖、太泊湖、企湖、大莲子糊、汉池湖的水鸟平均种类超过 15 种. 从 数量变化来看, $2000 / 2001-2012 / 2013$ 年越冬季呈波动变化, 其中 2001/2002 年和 2005/2006 年数量出现 2 个峰值, 总体来看, 水鸟数量维持在 30 万 40 万只. 其中单个越冬季数量最大值出现在大湖池 $(2001 / 2002$ 年), 水鸟数量为 143460 只 (图 2a).

从种类变化来看, 从 2003/2004-2005/2006 年水鸟种类呈明显增加趋势, 而在 2005/2006 年后, 种类数 变化比较平稳, 平均种类数维持在 $60 \sim 70$ 种. 其中, 单个越冬季种类最大值出现在常湖, 共调查到水鸟 42 种 (2006/2007 年) (图 2a).

从种群多样性来看, Shannon-Wiener 指数在从 2000/2001-2007/2008 年呈增加趋势, 此后, 物种多样性 指数呈下降趋势 (图 $2 b)$.
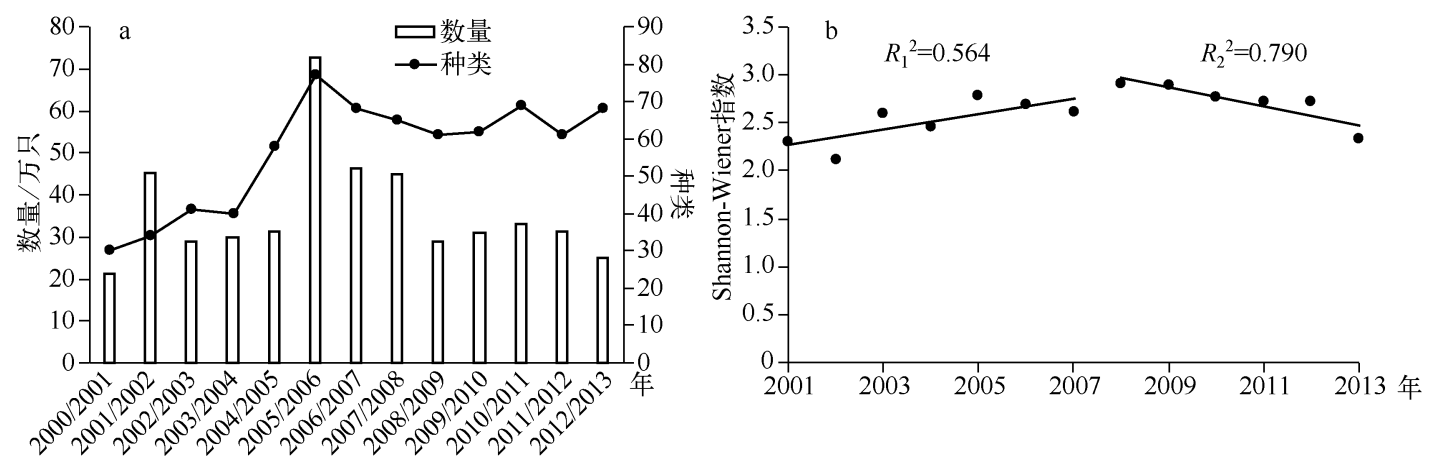

图 $22000 / 2001-2012 / 2013$ 年水鸟数量、种类变化 ( a) 和多样性变化 (b)

Fig. 2 Changes of number, species(a) and biodiversity (b) of waterfowl from 2000/2001 to 2012/2013

\section{2 子湖泊评价因子值}

子湖泊的数量种类综合因子间存在极显著差异 $(P<0.001$, 表 3$)$. 水鸟数量种类综合因子最高的为大 湖池, 其次为珠湖, 因子值都大于 0.75 . 数量种类综合因子值在 $0.50 \sim 0.75$ 之间的占 $19 \%, 0.25 \sim 0.50$ 之 间的占 $45 \%,<0.25$ 的占 $32 \%$. 数量种类综合因子最低的为南深湖, 湛公湖、晚湖、艾溪湖、苍湖也较低.

子湖泊的物种多样性综合因子、保护物种综合因子间存在显著差异 (分别 $P<0.001$ 和 $P<0.05$,表 3 ). 物种多样性因子在 $0.50 \sim 0.75$ 之间的占 $47 \%, 0.25 \sim 0.50$ 之间的占 $42 \%,<0.25$ 的占 0.11 . 其中物种多样 性综合因子最高的为太泊湖, 其次是大湖池、蚌湖、大莲子湖和新妙湖. 而南深湖、艾溪湖、南湖、湛公湖的物 种多样性综合因子较低. 保护物种综合因子整体较高, >0.75 的占 $44 \%, 0.50 \sim 0.75$ 之间的占 $27 \%, 0.25 \sim$ 0.50 之间的占 $19 \%,<0.25$ 的仅占 $10 \%$. 其中大湖池、插旗湖达到 1 , 而七里湖、谷山湖、湛公湖、艾溪湖、晚 湖、南深湖的值较低.

表 3 评价因子的均值、标准差和 Kruskal-Wallis 检验

Tab. 3 Mean, standard deviation and Kruskal-Wallis test of evaluation factors

\begin{tabular}{|c|c|c|c|c|c|c|c|c|}
\hline \multicolumn{3}{|c|}{ 数量种类综合因子 } & \multicolumn{3}{|c|}{ 保护物种综合因子 } & \multicolumn{3}{|c|}{ 物种多样性综合因子 } \\
\hline 均值 & 标准差 & $\chi^{2}$ & 均值 & 标准差 & $x^{2}$ & 均值 & 标准差 & $\chi^{2}$ \\
\hline 0.38 & 0.27 & $238.932 * * *$ & 0.67 & 0.37 & $228.738^{*}$ & 0.47 & 0.23 & $163.122^{* * *}$ \\
\hline
\end{tabular}

***代表 $P<0.001 ; *$ 代表 $P<0.05$. 


\section{3 子湖泊栖息地重要性的空间差异及年际变化趋势}

评价的子湖泊中, $60 \%$ 的子湖泊栖息地重要性值达 0.50 以上,鄱阳湖越冬水鸟栖息地总体处于比较重 要水平,但 62 个子湖泊间栖息地重要性在空间上呈现显著差异 $\left(\chi^{2}=185.948, d f=61, P=0\right)$. 其中重要性值 最高的为大湖池 $(0.868)$, 重要性值最小的为南深湖 ( 0.004$)$. 重要性值 $>0.75$ 的有 7 个子湖泊, 占 $11.29 \%$; 分别为大湖池 $(0.87)$ 、蚌湖 $(0.81)$ 、大莲子湖 $(0.81)$ 、珠湖 $(0.78)$ 、汉池湖 $(0.77)$ 、企湖 $(0.76) 、$ 太泊湖 ( 0.77$), 0.50 \sim 0.75$ 的有 30 个,占 $48.39 \%, 0.25 \sim 0.50$ 的有 20 个,占 $32.26 \%,<0.25$ 的有 5 个,占 $8.06 \%$ (图 3).

2000/2001-2012/2013 年以来,33 个子湖泊的重要性上升,其中,省级以上保护区内子湖泊有 14 个,其 他保护区有 9 个,保护区外有 10 个,占调查子湖泊总数的 $53.23 \%$. 在重要性上升的子湖泊中,保护区内子 湖泊所占比例为 $69.70 \%$,明显高于保护区外子湖泊. 其中显著上升的子湖泊有 7 个,占 $11.29 \%$, 分别是段 湖 $(F=6.026, P<0.05)$ 、黄金嘴 $(F=56.087, P<0.001)$ 、沙湖 $(F=4.902, P<0.05)$ 、输湖 $(F=7.402, P<$ $0.05) 、$ 象湖 $(F=25.968, P<0.001)$ 、战备湖 $(F=9.989, P<0.01)$ 、朱市湖 $(F=5.471, P<0.05)$ ( 图 3$)$.

$2000 / 2001-2012 / 2013$ 年以来, 重要性下降的子湖泊有 29 个,占调查子湖泊总数的 $46.77 \%$. 其中, 重 要性下降的湖泊中, 省级以上保护区有 4 个,其他保护区有 10 个,保护区外有 15 个. 在重要性下降的子湖泊 中,保护区内子湖泊所占比例为 $48.42 \%$ (省级以上保护区内子湖泊占 $13.79 \%$, 其他保护区内子湖泊占 $34.48 \%)$, 略低于保护区外子湖泊. 显著下降的子湖泊有 4 个, 占 $6.45 \%$, 分别是苶花池 $(F=8.983, P<$ $0.05)$ 、南溪湖 $(F=6.34, P<0.05)$ 、赛城湖 $(F=13.904, P<0.01)$ 、瑶湖 $(F=6.702, P<0.05)$ (图 3 ).
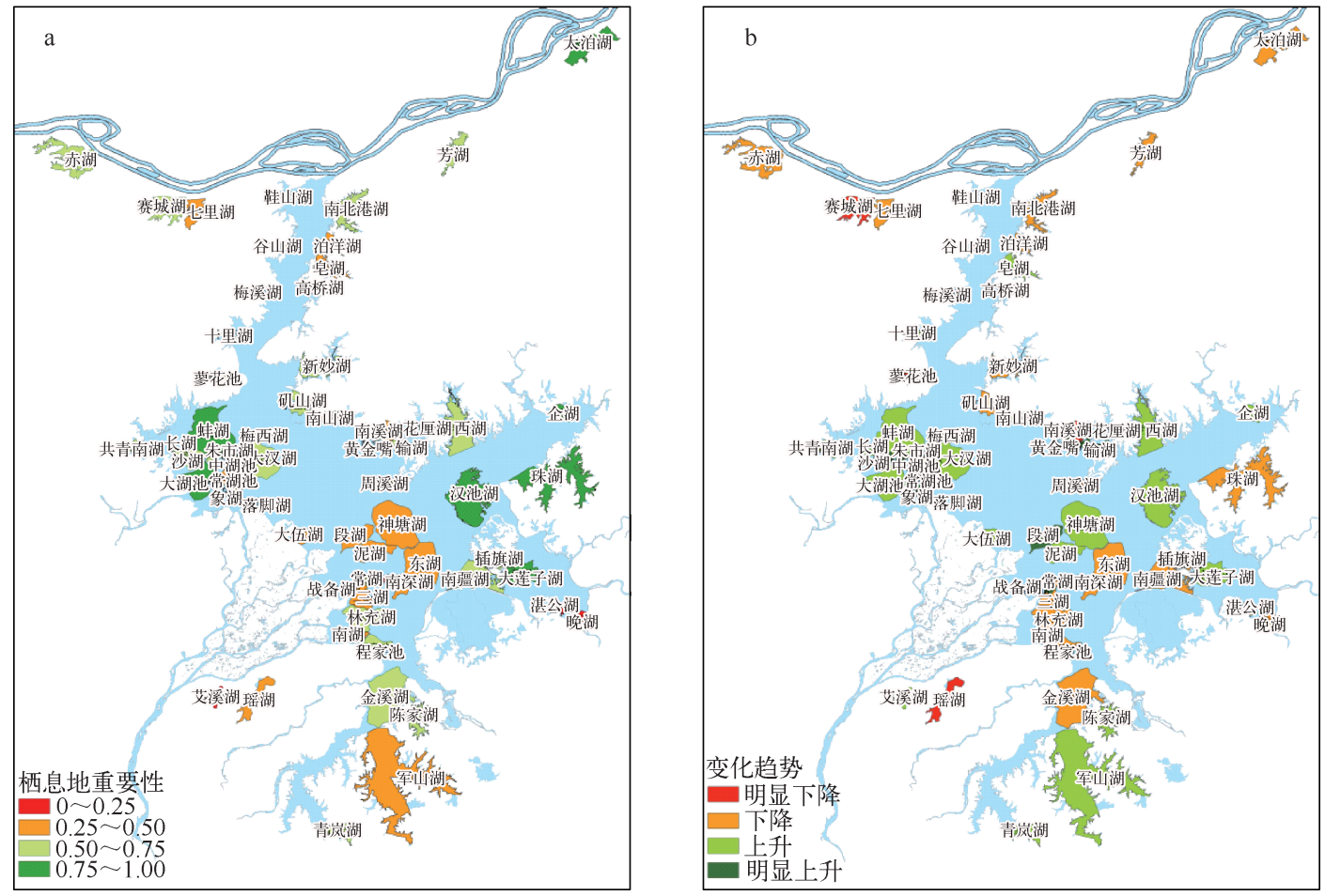

图 3 2000/2001-2012/2013 年栖息地重要性均值 (a) 和变化趋势 (b)

Fig. 3 Average(a) and variation trend(b) of habitat importance grade from 2000/2001 to 2012/2013

\section{4 保护等级对子湖泊重要性的影响}

不同保护等级子湖泊评价因子值之间存在显著差异 (表 4). 对比不同保护等级子湖泊的评价因子值, 自 然保护区内子湖泊的各评价因子都明显高于保护区外子湖泊,而省级以上自然保护区和其他保护区内子湖 泊间只有物种多样性综合因子存在显著差异 $(P<0.05)$, 其余评价因子则不存在显著差异 $(P>0.05)$. 从栖 
息地重要性等级来看,省级以上保护区和其他保护区内子湖泊不存在显著差异 $(P>0.05)$,但保护区内子湖 泊的栖息地重要性显著高于保护区外的子湖泊 $(P<0.05)($ 表 4$)$.

表 4 不同保护等级子湖泊的评价因子值 *

Tab. 4 Value of evaluation factors of sub-lakes under different protection levels

\begin{tabular}{|c|c|c|c|c|c|c|c|c|}
\hline \multirow{2}{*}{ 子湖泊等级 } & \multicolumn{2}{|c|}{ 数量种类综合因子 } & \multicolumn{2}{|c|}{ 保护物种综合因子 } & \multicolumn{2}{|c|}{ 物种多样性综合因子 } & \multicolumn{2}{|c|}{ 栖息地重要性 } \\
\hline & 均值 & 标准差 & 均值 & 标准差 & 均值 & 标准差 & 均值 & 标准差 \\
\hline 省级以上保护区 & $0.45^{\mathrm{a}}$ & 0.33 & $0.72^{\mathrm{a}}$ & 0.36 & $0.45^{b}$ & 0.24 & $0.58^{\mathrm{a}}$ & 0.28 \\
\hline 市 (县) 级保护区 & $0.37^{\mathrm{a}}$ & 0.23 & $0.71^{\mathrm{a}}$ & 0.33 & $0.51^{\mathrm{a}}$ & 0.21 & $0.58^{\mathrm{a}}$ & 0.24 \\
\hline 保护区外 & $0.34^{\mathrm{b}}$ & 0.26 & $0.60^{\mathrm{b}}$ & 0.41 & $0.45^{\mathrm{c}}$ & 0.23 & $0.50^{\mathrm{b}}$ & 0.29 \\
\hline
\end{tabular}

* 不同字母标注表明评价因子基于 Kruskal-Wallis 检验具有显著差异性, $P<0.05$. 数量种类综合因子 $\chi^{2}=9.969, P<0.01$; 保护物种综合因子 $\chi^{2}=8.464, P<0.05$; 物种多样性综合因子 $\chi^{2}=11.759, P<0.01$; 栖息地重要性 $\chi^{2}=10.656, P<0.01$.

\section{4 讨论}

\section{1 水鸟栖息地的评价方法}

以往的水鸟栖息地评价研究多依据气候、地形、食物等生境适宜性影响因素来反映湿地生境适宜性及 保护价值 ${ }^{[26-27]}$,评估结果能够反映栖息地的客观状态, 无法反映水鸟对栖息地的利用动态. 也有研究选择游 禽、涉禽及反映水鸟种群结构的因子或基于物种濒危等级的 IUCN 红色物种名录指数来评估栖息地质 量 ${ }^{[18,28]}$, 往往无法精确反馈种群结构的动态变化. 作为湿地结构与功能的重要指标, 水鸟物种多样性变化与 栖息地质量息息相关 ${ }^{[13-15]}$. 本文参考了 IBA 的标准,在考虑水鸟种群数量及保护价值的同时,将水鸟种群多 样性也作为衡量栖息地的质量的评价因子之一. 综合指标的应用不仅揭示了基于水鸟利用现状的栖息地重 要性,而且可以揭示水鸟栖息地重要性的变化动态.

\section{2 水鸟栖息地重要性的空间异质性}

水鸟对栖息地的选择主要受食物条件和可达性的影响 ${ }^{[12]}$, 而水文要素是影响鄱阳湖水鸟栖息地的重要 因素 ${ }^{[31]}$, 由于不同子湖泊在湿地水文过程及景观连接上的作用不同, 导致鄱阳湖水鸟栖息地重要性具有明 显的空间异质性. 鄱阳湖栖息地的重要性整体处于比较重要的水平,不同子湖泊间栖息地重要性却呈现显 著差异. 从数量种类综合因子来看, 大湖池、珠湖的数量种类综合因子明显高于其他湖泊, 这说明在鄱阳湖 水鸟种群呈现集中分布的特征. 种群的集中分布, 可能导致在资源的占有和分割中存在种内和种间竞争. 而 数量和种类在大湖池和珠湖的集中分布, 一方面可能是因为这 2 个湖泊提供的栖息地更为丰富, 也可能是 由于某些水鸟对栖息地选择的唯一性. 而保护物种综合因子的值均相对较高, 这说明鄱阳湖对珍稀濒危水 鸟的保护具有不可替代性. 2000/2001-2012/2013 年以来, 在鄱阳湖越冬的水鸟数量比较稳定甚至有所上 升 (图 2), 长江中下游其他湖泊的退化可能使一些原本在其他湖泊栖息的水鸟将鄱阳湖湿地做为新的栖息 地. 如升金湖沉水植被退化导致的食物资源鿒乏是 2008/2009 年越冬季升金湖鸿雁数量锐减的主要 原因 ${ }^{[3]}$.

\section{3 自然保护区在水鸟保护中的作用}

以水鸟与湿地为保护对象的自然保护区的建立, 加强了对水鸟监测与研究的能力. 本研究结果表明: 保 护区内栖息地的重要性明显高于保护区外,而保护区内子湖泊重要性上升的比例也明显高于保护区外子湖 泊 (表 4、图 3a), 且重要性显著上升的子湖泊均位于国家级和省级自然保护区内, 这说明保护区的保护管理 措施对水鸟栖息地起到了积极的作用. 建筑人工圩堤将子湖与鄱阳湖主湖隔断, 以及高密度的水产养殖活 动导致栖息地重要性值下降, 如苶花池, 2001/2002 年和 2002/2003 年越冬季, 栖息地的重要性值曾达到 0.75 ,而 2012/2013 年越冬季仅下降至 0.08 . 栖息地退化的主要原因是: 水产养殖的扩张侵占了水鸟的栖息 地,使得原来在此栖息的水鸟只能转而寻找新的栖息地. 而省级以下保护区内子湖泊存在管理权属界定不 清等问题, 这可能也是这些子湖泊水鸟栖息地功能下降的原因. 值得注意的是, 在这些子湖泊, 以往调查有 大量的水鸟,也是白鹤、白头鹤和东方白鹳等濒危物种的主要栖息地, 其栖息地功能重要性的下降说明其 
提供栖息地的功能退化,但其对水鸟而言仍具有重要意义,需要采取合理措施,进行栖息地恢复. 另外,珠 湖、企湖等重要性达到 0.75 以上的子湖泊并未覆盖在现有的保护区内,说明鄱阳湖在水鸟和湿地保护中仍 存在部分保护空缺.

\section{5 结论}

1 ）鄱阳湖 62 个子湖泊重要性值 $>0.75$ 的占 $11.29 \%, 0.50 \sim 0.75$ 的占 $48.39 \%$,水鸟栖息总体处于比 较重要的水平. 从不同评价因子来看,水鸟数量种类综合因子 $>0.50$ 的占 $68 \%$,物种多样性综合因子 $>0.50$ 的占 $47 \%$,保护物种综合因子值 $>0.50$ 的占 $71 \%$. 从变化趋势来看,重要性上升的子湖泊比例高于重要性 下降的子湖泊比例. 总体来看, 鄱阳湖栖息地功能重要性在增加,但变化趋势不明显; 水鸟数量和种类丰富, 对珍稀濒危水鸟保护的价值很高,充分发挥着国际重要湿地的功能.

2) 不同子湖泊的保护等级对鄱阳湖栖息地重要性产生明显影响,保护区对栖息地的保护和管理有效. 保护区内子湖泊重要性上升的比例明显高于保护区外子湖泊,而重要性下降的比例也略低于保护区外湖 泊. 不同保护等级子湖泊的评价因子值之间存在显著差异. 自然保护区内栖息地的重要性明显高于保护区 外. 但是从保护等级来看,省级以下保护区其栖息地重要性也很高,是水鸟重要的栖息地,但是其作为水鸟 栖息地功能却呈现出一定的退化,需要加大保护力度,并开展栖息地恢复措施. 另外,在保护区外的一些区 域仍存在部分保护空缺, 如珠湖、企湖等子湖泊.

致谢: 感谢鄱阳湖国家级自然保护区提供数据方面的支持,感谢张欢、贾亦飞、金杰锋在论文修改完善过程 中的建议.

\section{6 参考文献}

［1］农业部渔业局. 中国渔业统计年鉴 2012. 北京:中国农业出版社,2012.

[ 2 ] Fang JY, Wang ZH, Zhao SQ et al. Biodiversity changes in the lakes of the Central Yangtze. Frontiers in Ecology and the Environment, $2006,4(7): 369-377$.

[ 3 ] Fox AD, Cao L, Zhang Y et al. Declines in the tuber-feeding waterbird guild at Shengjin Lake National Nature Reserve, China- a barometer of submerged macrophyte collapse. Aquatic Conservation-Marine and Freshwater Ecosystems, 2011, 21 (1) : 82-91.

[ 4 ] Myers N, Mittermeier RA, Mittermeier CG et al. Biodiversity hotspots for conservation priorities. Nature, 2000 , 403:853858.

[ 5 ] Roberts CM, McClean CJ, Veron JEN et al. Marine biodiversity hotspots and conservation priorities for tropical reefs. Science, 2002, $295: 1280-1284$.

[ 6 ] Stapp P, Polis GA. Marine resources subsidize insular rodent populations in the Gulf of California, Mexico. Oecologia, $2003, \mathbf{1 3 4}(4): 496-504$.

[ 7 ] MacDonald LA, Farquharson N, Hall RI et al. Avian-driven modification of seasonal carbon cycling at a tundra pond in the hudson bay lowlands( Northern Manitoba, Canada). Arctic Antarctic and Alpine Research, 2014, 46(1):206-217.

[ 8 ] Bancroft GT, Gawlik DE, Rutchey K. Distribution of wading birds relative to vegetation and water depths in the northern Everglades of Florida, USA. Waterbirds, 2002, 25(3):265-277.

[ 9 ] Oja T, Alamets K, Parnamets H. Modelling bird habitat suitability based on landscape parameters at different scales. Ecological Indicators, 2005, 5(4):314-321.

[10 ] Baschuk MS, Koper N, Wrubleski DA et al. Effects of water depth, cover and food resources on habitat use of marsh birds and waterfowl in boreal wetlands of Manitoba, Canada. Waterbirds, 2012, 35 ( 1 ) : 44-55.

[11] McKnight SK, Hepp GR. Foraging-niche dynamics of Gadwalls and American coots in winter. Auk, 1998, 115(3):670683.

[12] Ma ZJ, Cai YT, Li B et al. Managing wetland habitats for waterbirds: An international perspective. Wetlands, 2010,30 (1) $: 15-27$.

[13 ] Kitchell JF, Schindler DE, Herwig BR et al. Nutrient cycling at the landscape scale: The role of diel foraging migrations by geese at the Bosque del Apache National Wildlife Refuge, New Mexico. Limnology and Oceanography, 1999, 44(3) : 
828-836.

[14] 张淑霞,董云仙,夏 峰. 湖泊生态系统的水鸟监测意义. 湖泊科学, 2011,23(2) : 155-162.

[15] Neave HM, Norton TW, Nix HA. Biological inventory for conservation evaluation II. Composition, functional relationships and spatial prediction of bird assemblages in southern Australia. Forest Ecology and Management, 1996, 85 ( 1 ): 123-148.

[16] Kingsford RT. Aerial survey of waterbirds on wetlands as a measure of river and floodplain health. Freshwater Biology, 1999, 41(2): 425-438.

[17] Konisky RA, Burdick DM, Dionne M et al. A regional assessment of salt marsh restoration and monitoring in the Gulf of Maine. Restoration Ecology, 2006, 14(4) :516-525.

［18］张淑萍, 张正旺,覃䈗燕. 模糊综合评价法在水鸟栖息地保护等级评价中的应用一一天津地区水鸟栖息地评价案 例. 北京师范大学学报: 自然科学版,2003,39(5):677-682.

[19] 刘 影, 范 娜, 于秀波等. 基于 RS 和 GIS 的鄱阳湖天然湿地边界确定及季节变化分析. 资源科学, 2010,32(11): 2239-2245.

[20］雷 声,张秀平,许小华. 鄱阳湖湿地植被秋冬季变化多源遥感监测分析. 人民长江,2011,42(11):60-63.

[21] 胡振鹏, 葛 刚, 刘成林等. 鄱阳湖湿地植物生态系统结构及湖水位对其影响研究. 长江流域资源与环境, 2010,19 (6) :597-605.

[22] Hoves JG, Bakewell D. Shore birds studies manual. Kuala Lumpur: AWB Publication, 1989.

[23] 马敬能,何芬奇, 菲利普斯. 中国鸟类野外手册. 长沙: 湖南教育出版社,2000.

[24] Onkal-Engin G, Demir Iand, Hiz H. Assessment of urban air quality in Istanbul using fuzzy synthetic evaluation. Atmospheric Environment, 2004, 38(23):3809-3815.

[25] Shen GQ, Lu YT, Wanget MN et al. Status and fuzzy comprehensive assessment of combined heavy metal and organo-chlorine pesticide pollution in the Taihu Lake region of China. Journal of Environmental Management, 2005, 76 (4): 355-362.

[26] 况润元, 周云轩,李 行等. 崇明东滩鸟类生境适宜性空间模糊评价. 长江流域资源与环境, 2009,18(3):229-233.

[27] Fornasari LE, Carli D, Bottoniet L et al. A method for establishing bird conservation value at a landscape level. Bird Conservation International, 1997,7 ( 1 ) :81-97.

[28 ] Butchart SHM, Scharlemann JPW, Evans MI et al. Protecting important sites for biodiversity contributes to meeting global conservation targets. PLoS One, 2012, $7(3): 1-7$.

[29] Bird Life International important bird areas in Asia: key sites for conservation. Cambridge, UK: Bird Life International, 2004.

[30］阎传海. 安徽省萧县皇藏峪自然保护区评价研究.农村生态环境,1997,13(4):12-15.

[31］夏少霞,于秀波,范 娜. 鄱阳湖越冬季候鸟栖息地面积与水位变化的关系. 资源科学,2010,32(11):2072-2078. 